\title{
Iran-Sanktionen: Optionen für die Europäische Union
}

Donald Trump nimmt nicht viel Rücksicht auf seine Verbündeten. Um das Vormachtstreben des Iran im Nahen Osten zu stoppen, verbietet er den Handel mit diesem Land nicht nur für US-Unternehmen, sondern für die europäischen Unternehmen gleich mit. Wer die Sanktionsmaßnahmen nicht befolgt, muss ab dem 6. August 2018 mit saftigen Geldbußen rechnen oder wird sogar vollständig von den US-amerikanischen Absatzmärkten verbannt. Die Empörung in Europa ist groß - und verständlich, denn schon die Aufkündigung des Atomabkommens durch die USA geschah gegen den Widerstand der europäischen Vertragspartner. Auch gegen die jetzt verhängte Verschärfung der Handelssanktionen hatten die europäischen Partner protestiert. Dass sich dieser transatlantische Disput vor dem Hintergrund der Schutzzölle der USA auf Stahl, Aluminium und möglicherweise mehr vollzieht, heizt die Debatte zusätzlich an. Europas Politiker wollen diese erneute Provokation aus Washington nicht tatenlos hinnehmen, sondern mutig und entschlossen Gegenmaßnahmen ergreifen.

Aber welche? Die betroffenen Unternehmen werden kaum aus eigenem Antrieb den USA die Stirn bieten. Denn der Export dorthin macht immerhin 8,7\% der deutschen Gesamtexporte aus, während der Anteil der Exporte in den Iran gerade einmal bei $0,3 \%$ liegt. Aber vielleicht könnte man die Unternehmen per Strafandrohung dazu bringen, die US-Sanktionen nicht zu befolgen. Für diesen Zweck haben zunächst der EU-Ratspräsident Donald Tusk und dann die Europäische Kommission angekündigt, die sogenannte Blocking Regulation reaktivieren zu wollen. Es handelt sich dabei um eine EU-Verordnung aus dem Jahr 1996, die sich gegen die Drittlandseffekte der damaligen US-Sanktionen gegen Kuba, den Iran und Libyen gerichtet hatte. Nach der Vorstellung der EU-Kommission sollen die neuen Iran-Sanktionen in die Verordnung aufgenommen werden. Dann wäre es verboten, diesen US-Vorschriften nachzukommen. In Deutschland ist die Blocking Regulation mit einem Bußgeld von 500000 Euro pro Verstoß bewehrt. Mit der Reaktivierung der Blocking Regulation ließe sich allerdings nur der Vollzug bereits geschlossener Verträge durchsetzen, aber kein Abschluss neuer Verträge erzwingen. Zudem würden gerade jene europäischen Unternehmen belastet, die ohnehin die Leidtragenden der US-Sanktionen sind. Schon 1996 wurden aus der Blocking Regulation keine Bußgelder verhängt, und das wäre heute wahrscheinlich genauso.

Aus Kreisen der Bundesregierung kam der Vorschlag, einen Fonds einzurichten, aus dem diejenigen Unternehmen entschädigt werden, die wegen der Fortführung ihrer Iran-Geschäfte mit US-Strafgeldern belegt würden. Damit würde allerdings den USBehörden quasi ein Selbstbedienungsladen eröffnet, denn es könnten ja beliebig hohe Strafgelder verhängt werden, die allesamt aus dem Fonds beglichen würden. Außerdem könnten die Unternehmen für entgangene US-Geschäfte Ersatz begehren, wenn sie zur Strafe für ihre Iran-Geschäfte vom US-Markt verbannt würden. Bei einem Volumen der gesamten deutschen Exporte in die USA von rund 100 Mrd. Euro pro Jahr könnten sich Entschädigungsansprüche ergeben, die schnell die Grenzen des Machbaren überschreiten würden.

Darüber hinaus könnte es für europäische Unternehmen künftig ausgesprochen schwierig werden, den mit dem Iranhandel verbundenen Zahlungsverkehr abzuwickeln. Denn aus Furcht vor US-Reaktionen trauen sich schon heute viele europäische Banken kaum noch, Finanzgeschäfte mit dem Iran zu tätigen. Die Deutsche Bank beispielsweise ist auf diesem Feld schon seit längerem äußerst zurückhaltend und

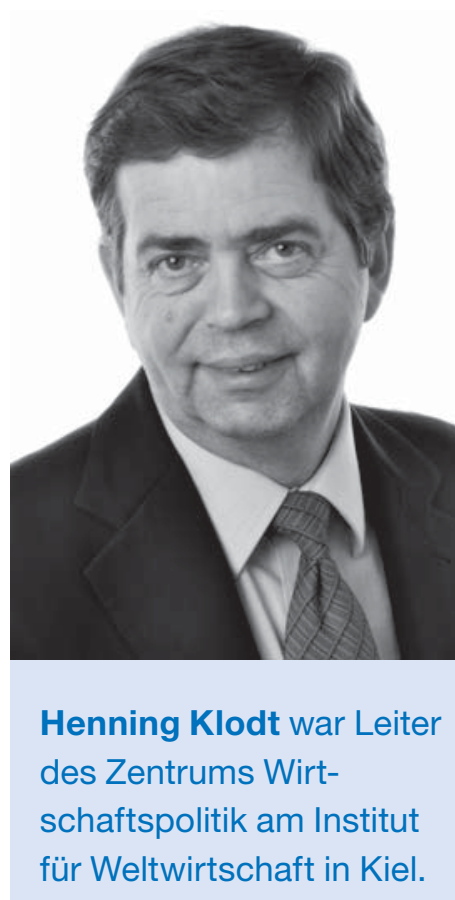


die Commerzbank hat ihre Iran-Aktivitäten mittlerweile vollkommen eingestellt. Jetzt haben auch die DZ Bank und mehrere Landesbanken, darunter die Bayerische Landesbank und die gerade erst privatisierte HSH Nordbank, verkündet, keine Finanzgeschäfte mehr mit dem Iran abwickeln zu wollen. So bleiben fast nur noch einige unentwegte Sparkassen und die im iranischen Staatsbesitz befindliche EuropäischIranische Handelsbank übrig. Insbesondere die Commerzbank weiß ein Lied davon zu singen, welche Folgen es haben kann, US-Sanktionen zu missachten. Sie wurde dafür 2015 mit einem Strafgeld in Höhe von 1,5 Mrd. US-\$ belegt. Noch härter traf es die BNP Paribas, der Strafzahlungen in Höhe von 9 Mrd. US-\$ auferlegt wurden, weil sie gegen damalige US-Sanktionen verstoßen hatte. Angesichts der ausgeprägten Dominanz der Wall Street auf den internationalen Finanzmärkten kann es sich praktisch keine europäische Bank erlauben, in den offenen Konflikt mit US-Behörden zu gehen. Trump hat also gute Aussichten, dass sich auch solche europäischen Unternehmen an den Sanktionen beteiligen werden, die selbst gar keine ausgeprägten Handelsinteressen in den USA haben.

Aber auch zu diesem Problemkreis hat die Europäische Kommission eine Idee. Sie hat jetzt einen Prüfauftrag an die Mitgliedstaaten erteilt, mit dem geklärt werden soll, inwieweit die nationalen Notenbanken dazu herangezogen werden können, Zahlungen an die iranische Nationalbank zu leisten, für welche die Geschäftsbanken nicht mehr zur Verfügung stehen. Dann könnte der Iran weiterhin sein Erdöl in Europa verkaufen und bekäme einen Zahlungskanal eröffnet, der ihm die Devisen verschafft, von denen die Trump-Administration inn eigentlich abschneiden wollte. Wie zu erwarten, sind die Notenbanken der EU-Mitgliedstaaten nicht begeistert. Sie verweisen unter anderem darauf, dass auch sie über die internationalen Finanzmärkte mit der Federal Reserve und der Wall Street verflochten sind und deshalb unnötige Konflikte mit den USA meiden möchten. Vor allem aber würden derartige Geschäfte weit über den gesetzlichen Auftrag der Zentralbanken hinausgehen. Falls dieser Vorstoß aus Brüssel wider alle Vernunft doch erfolgreich sein sollte, würden damit die Reputation und die Funktionsfähigkeit des europäischen Geld- und Währungssystems nachhaltig beschädigt.

Das Ergebnis all dieser Überlegungen ist zugegebenermaßen ein wenig unbefriedigend: Donald Trump kündigt ohne hinreichende Begründung den Atomvertrag mit dem Iran (immerhin hat der Iran nach Bekunden der anderen beteiligten Länder Deutschland, China, Russland, Frankreich und Großbritannien - sämtliche Auflagen des Vertrags buchstabengetreu erfüllt). Auf diesen Vertragsbruch stützen sich die verhängten US-Sanktionen, an denen sich die europäischen Unternehmen zu ihrem eigenen Schaden zwangsweise beteiligen müssen. Und den europäischen Regierungen fällt nichts ein, wie sie diesem dreisten Treiben Einhalt gebieten können.

Der oft zu hörende Hinweis, die EU sei mit ihrer Wirtschaftskraft durchaus auf Augenhöhe mit den USA und müsse sich deshalb solches Gebaren nicht bieten lassen, verkennt leider die Realität. Denn auf die Größe des Sozialprodukts kommt es hier nicht an. Auch nicht auf die uneinheitliche politische Stimme Europas. Entscheidend ist vielmehr die Dominanz der USA auf den internationalen Finanzmärkten, die sich auch mit EU-Reformen à la Macron nicht überwinden lässt. Hinzu kommt die vergleichsweise hohe Exportorientierung der europäischen Wirtschaft, die Strafmaßnahmen der USA für Europa ungleich schmerzhafter macht als entsprechende Vergeltungsmaßnahmen in die entgegengesetzte Richtung. So bleibt für die Politik nicht viel mehr als die Besinnung auf den Reinhold Niebuhr zugeschriebenen Wunsch, den Mut zu haben, Dinge zu ändern, die wir ändern können, die Gelassenheit aufzubringen, Dinge hinzunehmen, die wir nicht ändern können, und die Weisheit zu erlangen, das eine

Henning Klodt, Kiel

henning.klodt@freenet.de vom anderen zu unterscheiden. 